\section{Aplicaciones y proyecciones de los antagonistas del receptor de mineralocorticoides en el tratamiento de patologías cardiovasculares}

\author{
FERNANDO J. VERDUGO ${ }^{\mathrm{a}}$, FELIPE A. MONTELLANO ${ }^{\mathrm{a}}$, \\ JUAN E. CARREÑO ${ }^{1}$, ELISA T. MARUSIC ${ }^{1}$
}

\section{Mineralocorticoid receptor antagonists and therapeutic strategies of cardiovascular damage}

In recent years, much attention has focused on the role of aldosterone and mineralocorticoid receptors (MRs) in the pathophysiology of hypertension and cardiovascular disease. Patients with primary aldosteronism, in whom angiotensin II levels are low, have a higher incidence of cardiovascular complications than patients with essential hypertension. The Randomized Aldactone Evaluation Study (RALES) demonstrated that adding a non-specific MR antagonist, spironolactone, to a standard therapy that included angiotensin-converting enzyme (ACE) inhibitors, loop diuretics, and digoxin, significantly reduced morbidity and mortality in patients with moderate to severe heart failure. Similarly, the Eplerenone Post-Acute Myocardial Infarction Heart Failure Efficacy and Survival Study (EPHESUS) showed that the addition of a selective MR antagonist (ARM), eplerenone, to an optimal medical therapy reduces morbidity and mortality among patients with acute myocardial infarction complicated by left ventricular dysfunction and heart failure. These data suggest that aldosterone induces cardiac injury through activation of MRs and support the notion that MR blockade has beneficial effects on aldosterone-dependent cardiac injury, through mechanisms that cannot be simply explained by hemodynamic changes. Although, MRA are highly effective in patients with heart failure, the risk of hyperkalemia should not be overlooked. Serious hyperkalemia events were reported in some MRA clinical trials; however these risks can be mitigated through appropriate patient selection, dose selection, patient education, monitoring, and follow-up.

(Rev Med Chile 2014; 142: 61-71)

Key words: Aldosterone; Cardiovascular Diseases; Mineralocorticoid receptor antagonists; Spironolactone.
${ }^{1}$ Centro de Investigaciones

Biomédicas, Facultad de

Medicina, Universidad de los

Andes, Santiago, Chile.

anterno de Medicina, Universidad

de los Andes, Santiago de Chile.

Financiado por proyecto Fondo Ayuda investigación Universidad de los Andes FAI MED 004-

09 (JEC) y Premio Ulysses de

Investigación 2010 (FAM).

Recibido el 13 de junio de 2012, aceptado el 9 de enero de 2013

\section{Correspondencia a: \\ Dra. Elisa T. Marusic. \\ Teléfono: (562) 6181371. \\ emarusic@uandes.cl}

$\mathrm{D}$ urante la última década numerosos estudios han demostrado que los niveles elevados de aldosterona representan un factor de riesgo cardiovascular independiente de su acción renal. En efecto, pacientes con hiperaldosteronismo primario presentan una mayor incidencia de hipertensión arterial (HTA), insuficiencia cardiaca (IC), enfermedad coronaria y arritmias cardiacas, las que en el pasado eran atribuidas a la retención de sodio y aumento de volumen circulante ${ }^{1-3}$. La existencia del receptor de mineralocorticoides (RM) en vasos sanguíneos y tejido miocárdico explicaría que aumentos de la aldosterona circulantes ejerzan efectos deletéreos sobre el sistema cardiovascular. Dentro de estas acciones destacan procesos de remodelamiento y 
fibrosis cardiovascular, inflamación, aumento del estrés oxidativo, disfunción endotelial, alteración de la reactividad y el tono cardiovascular, y cambios en la electrofisiología cardiaca ${ }^{4}$. La Figura 1 resume los principales efectos que conlleva una elevación crónica de aldosterona. Cabe destacar que la acción deletérea sobre el sistema cardiovascular $(\mathrm{CV})$ es acentuada por una alta ingesta de sal e independiente de la presión arterial y de los niveles de angiotensina $\mathrm{II}^{4}$.

El uso de antagonistas del receptor de mineralocorticoides (ARM) ha permitido dilucidar en parte los efectos de aldosterona sobre el sistema cardiovascular y ha permitido el desarrollo de una importante línea terapéutica para diversas patologías. La presente revisión pretende recopilar algunos de los hallazgos más relevantes en relación al uso de ARM en pacientes con patologías cardiovasculares, haciendo hincapié en sus aplicaciones terapéuticas actuales y potenciales.

\section{Antagonistas del receptor de mineralocorticoides}

Existen dos fármacos incorporados en las guías clínicas actuales que inhiben competitivamente el RM: espironolactona y eplerenona. La primera posee elementos estructurales semejantes a la progesterona, lo que explica algunos efectos adversos de tipo progestagénicos y antiandrogénicos. Eplerenona es un antagonista más selectivo, con una menor afinidad por otros receptores hormonales.
Las principales características de ambos antagonistas se incluyen en Tabla $1^{5}$. El uso de metabolitos activos de espironolactona, canrenoato potásico (de administración endovenosa) y canrenona, ha sido reportado en ensayos clínicos, sin embargo, aún no existen guías que recomienden su utilización rutinaria ${ }^{6,7}$.

La hiperkalemia es el principal efecto adverso de ambos fármacos. Ensayos clínicos con uso de ARM en pacientes cardiópatas han reportado tasas bajas de hiperkalemia. Sin embargo, se han descrito cuadros de hiperkalemia secundarios al uso de ARM, causantes de aumento en hospitalizaciones y muertes súbitas ${ }^{8}$. Esto explicaría la subutilización en algunos casos de estos fármacos ${ }^{9,10}$. Las diferencias entre los ensayos clínicos y el uso habitual de ARM pueden ser atribuidas a lo restrictivo de los criterios de inclusión y exclusión empleados en dichos estudios, algo por lo demás común a todo ensayo clínico. La utilización de beta bloqueadores e inhibidores de la enzima convertidora de angiotensina (IECA) o antagonistas del receptor de angiotensina (ARA2) en etapas tempranas dentro del esquema terapéutico de pacientes cardiópatas también podría influir en esta discrepancia ${ }^{8}$. Para disminuir el riesgo de hiperkalemia, las guías clínicas actuales sugieren que pacientes con indicación de ARM inicien la terapia con una kalemia $<5 \mathrm{mEq} / \mathrm{L}$ y creatinina $\leq 2,5 \mathrm{mg} / \mathrm{dl}$ en hombres $\mathrm{y} \leq 2,0 \mathrm{mg} / \mathrm{dl}$ en mujeres, habiendo suspendido suplementos de potasio. Deben realizase controles periódicos de la función renal y electrolitos plasmáticos, especialmente en pacientes tratados

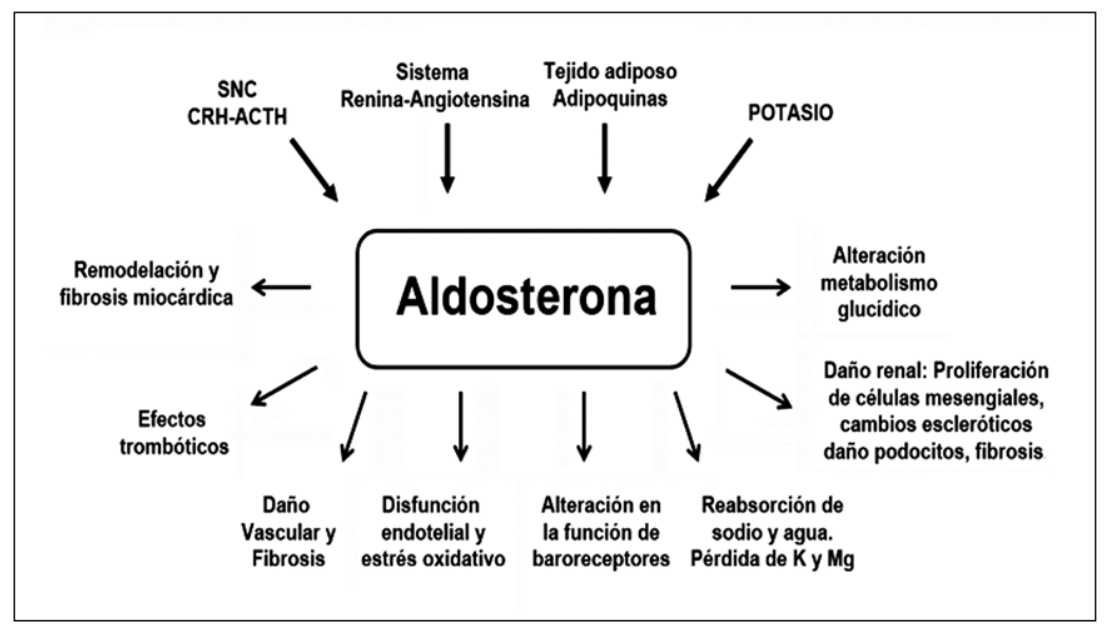

Figura 1. Principales efectos de aldosterona en el organismo. SNC: Sistema Nervioso Central. $\mathrm{CRH}$ : Hormona liberadora de corticotropina. ACTH: Hormona adrenocorticotrópica. K: Potasio. Mg: Magnesio. 
Tabla 1. Propiedades farmacológicas de antagonistas del receptor de mineralocorticoides

\begin{tabular}{|c|c|c|c|c|}
\hline & \multicolumn{2}{|c|}{ Espironolactona } & \multicolumn{2}{|l|}{ Eplerenona } \\
\hline Antagonismo & \multicolumn{2}{|l|}{ No selectivo } & \multicolumn{2}{|l|}{ Selectivo } \\
\hline Biodisponibilidad oral & \multicolumn{2}{|l|}{$\geq 90 \%$} & \multicolumn{2}{|l|}{$67 \%$} \\
\hline Unión a proteína & \multicolumn{2}{|l|}{$90 \%$} & \multicolumn{2}{|l|}{$50 \%$} \\
\hline Metabolismo & \multicolumn{2}{|l|}{ Hepático y renal } & \multicolumn{2}{|l|}{ Hepático } \\
\hline Metabolitos activos & \multicolumn{2}{|l|}{ Sí } & \multicolumn{2}{|l|}{ No } \\
\hline Excreción & Renal (47-51\%) & Biliar (35-41\%) & Renal (67\%) & Biliar (32\%) \\
\hline Vida media (horas) & Compuesto: 1,4 & Metabolitos: 14-22 & $4-6$ & \\
\hline Efectos adversos & \multicolumn{2}{|c|}{ Hiperkalemia, disfunción eréctil, ginecomastia, mastalgia } & \multicolumn{2}{|c|}{ Hiperkalemia, dolor abdominal, diarrea } \\
\hline Indicaciones clínicas & \multicolumn{2}{|c|}{ IC NYHA III-IV, HTA e HAP } & \multicolumn{2}{|c|}{ IC post IAM, HTA } \\
\hline Costo tratamiento & \multicolumn{2}{|l|}{ US\$ 0,35/día } & \multicolumn{2}{|l|}{ US\$ 3,75/día } \\
\hline Disponible en Chile & \multicolumn{2}{|l|}{ Sí } & \multicolumn{2}{|l|}{ No } \\
\hline
\end{tabular}

con los fármacos antes mencionados. Los efectos adversos progestagénicos y antiandrogénicos (ginecomastia, alteraciones menstruales, etc.) en algunos pacientes tratados con dosis $>50 \mathrm{mg}$ de espironolactona no revisten una complicación vital pero sí son una importante causa de incumplimiento del tratamiento, debiendo preferirse eplerenona en estos casos.

\section{ARM en insuficiencia cardiaca}

Ensayos clínicos con dosis bajas de ARM en pacientes con IC severa o disfunción sistólica post infarto agudo al miocardio, han demostrado una reducción significativa de la morbimortalidad ${ }^{11,12}$. El estudio RALES (Randomized Aldactone Evaluation Study, $n=1.663$ ) fue el primero en señalar a nivel clínico la importancia de la aldosterona en la fisiopatología de la IC severa, al demostrar en pacientes con IC con clasificación funcional III-IV según la New York Heart Association que una dosis de $25 \mathrm{mg}$ de espironolactona, unida al tratamiento estándar, disminuía en $30 \%$ la mortalidad y en 35\% la hospitalización por complicaciones cardiacas ${ }^{11}$. Posteriormente, el estudio EPHESUS (Eplerenone Post-Acute Myocardial Infarction Heart Failure Efficacy and Survival Stu$d y, n=6.632$ ) demostró que adicionar $40 \mathrm{mg}$ de eplerenona a la terapia convencional en pacientes post infarto agudo al miocardio (post-IAM) con disfunción sistólica, definida como fracción de eyección de ventrículo izquierdo $(F E V I) \leq 40 \%$, disminuía en $15 \%$ la mortalidad global, en $17 \%$ la mortalidad cardiovascular y en $21 \%$ la muerte súbita $^{12}$. Meta-análisis recientes han señalado que el uso de ARM se asocia a un aumento de la FEVI $(\overline{\mathrm{X}}=3,1 \% ; \mathrm{p}<0,05)$, y una disminución significativa del riesgo de muerte súbita y taquicardias ventriculares $^{13,14}$.

Cabe destacar que la efectividad clínica que han demostrado ambos fármacos no puede explicarse únicamente por su efecto diurético y antihipertensivo $^{11,12}$. Clínicamente, se ha demostrado que los pacientes con IC presentan niveles plasmáticos elevados de marcadores de estrés oxidativo, inflamación y síntesis de colágeno, los cuales se han asociado al daño tisular ${ }^{15}$. Además, se demostró una correlación positiva entre la concentración plasmática de algunos marcadores de dichos procesos y la concentración de aldosterona ${ }^{15}$. Estudios experimentales han señalado que los ARM son capaces de revertir los efectos deletéreos de aldosterona a nivel cardiaco disminuyendo significativamente la generación de especies reactivas de oxígeno, procesos inflamatorios y el remodelamiento de la matriz extracelular ${ }^{16-18}$.

Considerado el éxito de los ensayos clínicos RALES y EPHESUS, una serie de estudios posteriores han evaluado el efecto de ARM sobre la morbimortalidad de pacientes con IC leve a moderada (NYHA I-II) bajo terapia convencional. En el estudio EMPHASIS-HF (Eplerenone in Mild Patients Hospitalization and Survival Study in Heart Failure, 
Antagonistas del receptor de mineralocorticoides en patologías cardiovasculares - F. J. Verdugo et al

Tabla 2. Principales estudios clínicos de antagonistas del receptor de mineralocorticoides en pacientes con insuficiencia cardiaca

\begin{tabular}{|c|c|c|c|c|}
\hline Estudio & RALES ${ }^{11}$ & EPHESUS ${ }^{12}$ & EMPHASIS-HF ${ }^{19}$ & TOPCAT $^{30}$ \\
\hline Tipo & $\mathrm{RCT}$ & $\mathrm{RCT}$ & $\mathrm{RCT}$ & $\mathrm{RCT}$ \\
\hline Población & IC NYHA III-IV & IC post-IAM & IC NYHA II & IC NYHA II-IV \\
\hline FEVI (\%) & $<35$ & $<40$ & $<35$ & $>45$ \\
\hline $\mathrm{N}^{\circ}$ & 1.663 & 6.642 & 2.737 & $3.515^{*}$ \\
\hline Endpoint principal & Mortalidad & $\begin{array}{l}\text { MCV } \\
\text { Hosp. por IC }\end{array}$ & $\begin{array}{l}\text { MCV } \\
\text { Hosp. por IC }\end{array}$ & $\begin{array}{l}\text { MCV } \\
\text { Hosp. por IC }\end{array}$ \\
\hline ARM & Espiro & Eplerenona & Eplerenona & Espiro \\
\hline Dosis (mg/día) & 26 & 50 & $\leq 50$ & $\leq 45$ \\
\hline \multicolumn{5}{|c|}{ Reducción de riesgo } \\
\hline Mortalidad general & $\begin{array}{l}\downarrow 30 \% \\
(p<0,001)\end{array}$ & $\begin{array}{l}\downarrow 15 \% \\
(p<0,008)\end{array}$ & $\begin{array}{l}\downarrow 24 \% \\
(p<0,008)\end{array}$ & En curso \\
\hline MCV & $\begin{array}{l}\downarrow 32 \% \\
(p<0,001)\end{array}$ & $\begin{array}{l}\downarrow 17 \% \\
(p<0,005)\end{array}$ & $\begin{array}{l}\downarrow 37 \% \\
(p<0,01)\end{array}$ & En curso \\
\hline Hosp. por IC & $\begin{array}{l}\downarrow 35 \% \\
(p<0,001)\end{array}$ & $\begin{array}{l}\downarrow 23 \% \\
(p<0,002)\end{array}$ & $\begin{array}{l}\downarrow 42 \% \\
(p<0,001)\end{array}$ & En curso \\
\hline
\end{tabular}

(RCT: Ensayo clínico randomizado controlado. MCV: Mortalidad cardiovascular. Hosp: Hospitalización. Espiro: Espironolactona. *Esperado). Agregar significado de las restantes siglas, RCT, IC, FVEI, NYHA

$n=2.737)$ con 21 meses de seguimiento y $50 \mathrm{mg}$ de eplerenona añadidos al tratamiento estándar de IC moderada, se observó una reducción de $24 \%$ del riesgo de mortalidad cardiovascular y de $29 \%$ de las hospitalizaciones por complicaciones cardiacas $^{19}$. Ensayos clínicos de menor tamaño muestral en pacientes con IC leve tratados con ARM han demostrado un aumento significativo de la FEVI y una disminución de la expresión de marcadores de remodelamiento miocárdico ${ }^{20-23}$. Tabla 2 resume los principales ensayos clínicos realizados.

Las guías clínicas actuales recomiendan el uso de ARM en pacientes que presentan una IC NYHA III-IV, o post-IAM con FEVI $<40 \%$, con un nivel de recomendación $1 \mathrm{~B}^{6,7}$. Es esperable que amplíen sus recomendaciones a pacientes con IC con FEVI $<40 \%$, independiente de su capacidad funcional.

\section{ARM en insuficiencia cardiaca diastólica}

La activación del RM induce hipertrofia y fibrosis miocárdica de forma temprana ${ }^{17,18}$, ambos fenómenos responsables de una disminución de la compliance e incapacidad de relajación ventricular observada en la insuficiencia cardiaca diastólica (ICD). Ensayos clínicos en pacientes con IC y disfunción sistólica muestran mejoras de la función diastólica dentro de sus hallazgos ecocardiográficos ${ }^{23}$. Estudios experimentales en ratas hipertensas que desarrollan ICD han demostrado un aumento de la expresión del RM miocárdica ${ }^{24}$. En el mismo modelo, se señaló que los ARM son capaces de inhibir el remodelamiento miocárdico y prevenir el desarrollo de ICD ${ }^{24}$. Ensayos clínicos, de pequeño tamaño muestral, en pacientes con ICD que recibieron junto al tratamiento convencional canrenona, espironolactona o eplerenona demostraron una mejora de los parámetros ecocardiográficos de disfunción diastólica, con mejoras en la sintomatología y la calidad de vida, y sin modificar significativamente los valores de masa ventricular, presión arterial o capacidad aeróbica respecto a grupos control ${ }^{25-29}$. Además se encontró una disminución significativa de marcadores de síntesis del colágeno en el grupo tratado con eplerenona por 6 meses $^{29}$. Estos datos apoyan que, al menos en el corto plazo, los ARM mejoran la función diastólica miocárdica y modulan la progresión de la ICD.

El efecto que el tratamiento con ARM pudiese tener sobre la morbimortalidad a largo plazo de pacientes con ICD está siendo investigado 
en ensayo clinico con distribución aleatoria y multicéntrico TOPCAT (Treatment Of Preserved Cardiac function heart failure with an Aldosterone anTagonist) cuyo outcome primario incluye el paro cardiaco abortado, mortalidad cardiovascular y hospitalizaciones por IC ${ }^{30}$. Además hay en desarrollo otros 2 ensayos en pacientes con ICD, los que evalúan el uso de ARM sobre la capacidad aeróbica y marcadores de daño cardiovascular ${ }^{27,31}$ (Tabla 3).

\section{ARM en infarto agudo al miocardio}

Se ha descrito que la activación del receptor de mineralocorticoides por aldosterona produce empeoramiento de la función contráctil y metabólica del miocardio sometido a isquemia ${ }^{32}$. Esta situación se ve agravada además por la existencia de producción intracardiaca de aldosterona frente a isquemia ${ }^{32}$. Estudios prospectivos han reportado que pacientes con infarto agudo al miocardio (IAM) que cursan con niveles elevados de aldosterona al ingreso hospitalario exhiben un mayor riesgo de desarrollar IC, arritmias ventriculares y muerte cardiovascular ${ }^{33}$.

Estudios experimentales han señalado que el bloqueo del RM inmediatamente posterior a un IAM impide el remodelamiento eléctrico ${ }^{34}$, modula la respuesta inflamatoria secundaria a isquemia ${ }^{35}$, y logra un efecto cardioprotector ante el daño por reperfusión ${ }^{32}$. Estudios clínicos han sugerido un beneficio de la administración precoz de ARM en pacientes post-IAM. Un ensayo clínico con distribución aleatoria en pacientes sometidos a angioplastía $(\mathrm{n}=134)$, demostró que el uso de un bolo de $200 \mathrm{mg}$ de canrenoato inmediatamente posterior a revascularización, con administración de espironolactona desde el primer día, previene parcialmente el remodelamiento miocárdico postIAM asociado a una disminución de un marcador de síntesis colágena ${ }^{36}$. Una cohorte en pacientes post-IAM sin IC tratados con el mismo protocolo terapéutico reportó una reducción de arritmias ventriculares y paros cardiacos en tratados versus control histórico ${ }^{37}$.

Se encuentra en desarrollo ALBATROSS (Aldosterone Blockade Early After Acute Myocardial Infarction, $n=1.600$ ), ensayo clínico aleatorio, doble ciego y multicéntrico que busca determinar la efectividad del tratamiento con $200 \mathrm{mg}$ de canrenoato endovenoso sumado a $25 \mathrm{mg}$ de espironolactona por vía oral sobre la morbimortalidad de pacientes cursando un $\mathrm{IAM}^{38}$. El estudio incluirá a pacientes dentro de las primeras $72 \mathrm{~h}$ de evolución de $\operatorname{IAM}^{38}$ (Tabla 4).

Tabla 3. Principales estudios clínicos de antagonistas del receptor de mineralocorticoides en pacientes con insuficiencia cardiaca diastólica

\begin{tabular}{|c|c|c|c|c|c|c|}
\hline Estudio & Tipo & Población & $\mathbf{n}$ & $\begin{array}{l}\text { Endpoint } \\
\text { principal }\end{array}$ & ARM & $\begin{array}{l}\text { Resultados } \\
\text { (post vs pretratamiento) }\end{array}$ \\
\hline $\begin{array}{l}\text { Grandi } \\
\text { et al. }{ }^{25}\end{array}$ & $\mathrm{RT}$ & $\begin{array}{l}\mathrm{HTA}+\mathrm{ICD} \\
\mathrm{FEVI}>50 \%\end{array}$ & 34 & Función diastólica VI & $\begin{array}{l}\text { Canrenona } \\
25 \mathrm{mg} / \mathrm{d}\end{array}$ & $\begin{array}{l}E / A 1,11 \pm 0,24 \text { vs } 0,86 \pm 0,22(p<0,01) \\
T D 163 \pm 34 \text { vs } 209 \pm 38(p<0,001)\end{array}$ \\
\hline $\begin{array}{l}\text { Mottram } \\
\text { et al. }{ }^{26}\end{array}$ & $\mathrm{RCT}$ & $\begin{array}{l}\text { NYHA II } \\
\text { FEVI }>50 \%\end{array}$ & 30 & Función diastólica VI & $\begin{array}{l}\text { Espiro } \\
25 \mathrm{mg} / \mathrm{d}\end{array}$ & $\begin{array}{l}\text { E/A } 0,81 \pm 0,17 \text { vs } 0,79 \pm 0,12(\mathrm{NS}) \\
\text { TD } 254 \pm 23 \text { vs } 278 \pm 23(p=0,022)\end{array}$ \\
\hline $\begin{array}{l}\text { Daniel } \\
\text { et al. }{ }^{27}\end{array}$ & OLT & $\begin{array}{l}\text { NYHA } \|-I I I \\
\text { FEVI }>50 \%\end{array}$ & 11 & $\begin{array}{l}\text { Tolerancia ejercicio. } \\
\text { Función diastólica VI } \\
\text { Calidad de vida }\end{array}$ & $\begin{array}{l}\text { Espiro } \\
25 \mathrm{mg} / \mathrm{d}\end{array}$ & $\begin{array}{l}\mathrm{MVO}_{2} \uparrow 8,3 \%(p=0,001) \\
\mathrm{E}^{\prime} \downarrow 25 \%: 8,1 \pm 1,5 \text { vs } 10,8 \pm 5,6(p=0,02) \\
\text { Cambio NYHA III a II }(p=0,004)\end{array}$ \\
\hline $\begin{array}{l}\text { Roongsritong } \\
\text { et al. }{ }^{28}\end{array}$ & $\mathrm{RCT}$ & $\begin{array}{l}\text { ICD leve } \\
\text { FEVI }>50 \%\end{array}$ & 26 & Función diastólica VI & $\begin{array}{l}\text { Espiro } \\
25 \mathrm{mg} / \mathrm{d}\end{array}$ & $\begin{array}{l}E / A 0,84 \pm 0,19 \text { vs } 0,71 \pm 0,08(p=0,02) \\
\text { TD } 230 \pm 54,7 \text { vs } 285,5 \pm 73,1(p<0,04)\end{array}$ \\
\hline $\begin{array}{l}\text { Deswal } \\
\text { et al. } .^{29}\end{array}$ & $\mathrm{RCT}$ & $\begin{array}{l}\text { NYHA II-III } \\
\text { FEVI }>50 \%\end{array}$ & 44 & TC6m & $\begin{array}{l}\text { Eplerenona } \\
50 \mathrm{mg} / \mathrm{d}\end{array}$ & $\begin{array}{l}\text { TC6m (NS) } \\
\text { E/E' } 10,88 \pm 4,39 \text { vs } 12,65 \pm 4,42(p<0,01)\end{array}$ \\
\hline
\end{tabular}

(RT: Ensayo clínico randomizado no controlado. RCT: Ensayo clínico randomizado controlado. OLT: Ensayo clínico abierto no controlado. VI: Ventrículo izquierdo. TC6m: Test de caminata de 6 min. Espiro: Espironolactona. E/A: relación entre las onda E (llenado rápido del $\mathrm{VI}$ ) y la onda $\mathrm{A}$ (llenado $\mathrm{VI}$ secundario a contracción auricular). TD: Tiempo de desaceleración. $\mathrm{MVO}_{2}$ : Máximo consumo de oxígeno. E/E': relación entre las ondas $\mathrm{E}$ (llenado rápido del $\mathrm{VI}$ ) y la onda E' (doppler tisular del anillo mitral). NS: No significativo). 
Tabla 4. Principales estudios clínicos de antagonistas del receptor de mineralocorticoides en pacientes con infarto agudo al miocardio sin insuficiencia cardiaca

\begin{tabular}{|c|c|c|c|}
\hline Estudio & Hayashi et al ${ }^{36}$ & Beygui et $a^{37}$ & ALBATROSS ${ }^{38}$ \\
\hline Tipo & $\mathrm{RCT}$ & $\mathrm{CPCH}$ & $\mathrm{RCT}$ \\
\hline $\mathrm{N}^{\circ}$ & 134 & 154 vs 623 & $1.600^{*}$ \\
\hline Endpoint principal & Remodelamiento VI & Mortalidad, PCR, AV. & Mortalidad, PCR, AV, IC. \\
\hline Población & $\mathrm{IAM}+\mathrm{PCl}$ & $\mathrm{IAM}+\mathrm{PCl}$ & IAM \\
\hline ARM & $\begin{array}{l}\text { CK } 200 \text { mg ev, } \\
\text { Espiro } 25 \text { mg/día }\end{array}$ & $\begin{array}{l}\text { CK } 200 \text { mg ev, } \\
\text { Espiro } 25 \text { mg/día }\end{array}$ & $\begin{array}{l}\text { CK } 200 \text { mg ev, } \\
\text { Espiro } 25 \text { mg/día }\end{array}$ \\
\hline FEVI (\%) & $\begin{array}{l}7,2 \pm 0,7 \text { vs } 4,46 \pm 0,8 \\
(p<0,05)\end{array}$ & $\mathrm{NE}$ & \\
\hline VFDVI $\left(\mathrm{mL} / \mathrm{m}^{2}\right)$ & $\begin{array}{l}4,0 \pm 2,1 \text { vs } 19,2 \pm 3,2 \\
(p<0,001)\end{array}$ & NE & \\
\hline \multicolumn{4}{|c|}{ Reducción de riesgo } \\
\hline Mortalidad general & NE & NS & En curso \\
\hline AV & NE & $\downarrow 77 \%(p<0,0001)$ & En curso \\
\hline PCR & NE & $\downarrow 61 \%(p<0,05)$ & En curso \\
\hline
\end{tabular}

(RCT: Ensayo clínico randomizado controlado. CPCH: Cohorte prospectivo con control histórico. VI: Ventrículo Izquierdo. PCR: Paro cardiaco/cardiorrespiratorio reanimado. AV: arritmias ventriculares. IC: Desarrollo o progresión de insuficiencia cardiaca. PCl: Angioplastía. CK: Canrenoato potásico. Espiro: Espironolactona. VFDVI: Volumen de fin de diástole del ventrículo izquierdo. *Esperado. NE: No evaluado. NS: No significativo).

\section{ARM en fibrilación auricular}

Durante la última década, se ha intentado desarrollar una terapia causal ("upstream therapy") para la fibrilación auricular (FA), la que tendría como objetivo suprimir las rutas que sirven de sustrato para la generación de dicha arritmia ${ }^{39}$. Parte de la atención se ha centrado en el papel que cumpliría la activación del sistema renina angiotensina aldosterona (SRAA) sobre el remodelamiento auricular, así como el posible bloqueo del SRAA. Si bien la mayoría de los trabajos existentes se centran en el rol deletéreo de angiotensina II y el uso de IECA o ARA2, estudios recientes señalan la participación de aldosterona y del RM en la fisiopatología de esta arritmia. Investigaciones en animales han demostrado que aldosterona es capaz de inducir fibrosis auricular, hipertrofia de los cardiomiocitos y producir alteraciones de la conducción que contribuyen al desarrollo de arritmias auriculares ${ }^{40}$. Esto podría deberse, al menos en parte, a un aumento de la expresión génica del RM en aurícula izquierda en este tipo de pacientes $^{41,42}$. Estudios epidemiológicos han señalado que pacientes con HAP presentan un riesgo 12 veces mayor de desarrollar FA en comparación a controles pareados por presión arterial². Además, se han reportado aumentos en los niveles de aldosterona en pacientes con FA persistente y permanente, versus pacientes con ritmo sinusal o convertidos mediante cardioversión eléctrica ${ }^{43-45}$. La FA podría entonces activar mecanismos de producción intracardiaca de aldosterona, de manera similar a lo descrito previamente en modelos de $\mathrm{IC}^{46}$, que potencie el desarrollo del sustrato de esta arritmia.

Estudios experimentales muestran que el tratamiento con ARM es capaz de disminuir la fibrosis auricular, la dilatación auricular y la capacidad de inducción de arritmias auriculares ${ }^{47-49}$. Estos datos han sido corroborados en parte por ensayos clínicos realizados en pacientes con IC que demuestran una menor tendencia a desarrollar FA y una disminución significativa del volumen auricular al ser tratados con $\mathrm{ARM}^{19,22,50}$. En términos de morbilidad asociada, una cohorte retrospectiva en pacientes con FA y cardiopatía estructural concomitante ( $\mathrm{n}=83,74 \%$ FA paroxística), indicó que el grupo tratado con espironolactona por 3 meses o más $(\mathrm{n}=23)$ presentó una menor tasa de hospitalizaciones o necesidad de cardioversión 
eléctrica en comparación a pacientes no tratados $(22 \% \text { vs } 53 \%, p<0,009)^{51}$. Fuera del contexto de cardiopatías concomitantes, el estudio SPIR-AF (Spironolactone in Atrial Fibrillation, $n=164$ ), estudio aleatorio llevado a cabo en pacientes con historia de FA paroxística sintomática reportó la disminución significativa de episodios sintomáticos, por electrocardiografía, en los pacientes tratados con espironolactona versus los pacientes de los restantes grupos, a los 3, 6, 9 y 12 meses de iniciado el tratamiento ( $\mathrm{p}<0,001)$, no encontrándose diferencias significativas de frecuencia cardiaca, presión arterial ni cambios de la kalemia entre los grupos tratados con espironolactona ${ }^{52,53}$.

Los resultados actuales sugieren una potencial utilidad terapéutica de los ARM en pacientes con FA paroxística, particularmente en pacientes con cardiopatía y/o hipertensión arterial concomitante. Falta realizar ensayos clínicos de mayor envergadura que evalúen el potencial impacto sobre la morbimortalidad que esta terapia tendría en todos los estadios de FA.

\section{ARM en hipertensión arterial}

La activación del RM ha demostrado tener un claro papel en la etiopatogenia de la HTA. El hiperaldosteronismo constituye la forma más prevalente de HTA secundaria, correspondiente a alrededor de $15 \%$ de la población hipertensa al utilizar como método de screening la razón entre concentración de aldosterona plasmática y actividad de renina plasmática ${ }^{1}$. En pacientes con hipertensión arterial resistente (HTAR), definida como HTA que requiere $\geq 3$ antihipertensivos en dosis óptimas y un diurético, la prevalencia de HAP es de $14-23 \%{ }^{3}$. El Framingham Offspring Study $(\mathrm{n}=$ 1.688) demostró que pacientes normotensos con aldosterona normales, pero que durante el período estudiado ( 4 años) presentaron un aumento de la aldosteronemia dentro del rango fisiológico, con valores en el cuartil superior respecto al cuartil más bajo, tuvieron un riesgo aumentado de presentar HTA durante el estudio $(\mathrm{RR}=1,61)^{53}$. Basados en la efectividad clínica de los ARM en subpoblaciones de pacientes hipertensos con niveles fisiológicos de aldosterona (obesos, diabéticos, nefropatías), algunos autores han sugerido una sobrexpresión o hipersensibilidad del RM en estos pacientes $^{54}$.
Espironolactona posee un reconocido papel al combinarse con otras drogas en el manejo de la HTAR. Una revisión sistemática de la literatura incluyendo 5 ensayos clínicos no controlados, han señalado una reducción promedio de 22/10 $\mathrm{mmHg}$ al utilizar espironolactona en dosis bajas como cuarto antihipertensivo ${ }^{59}$. Un ensayo no controlado con eplerenona reportó resultados similares ${ }^{60}$. En el ensayo randomizado ASPIRANT (Addition of Spironolactone in Patients With Resistant Arterial Hypertension, $n=117$ ), los pacientes con HTAR fueron tratados con $25 \mathrm{mg} /$ día de espironolactona, reportándose una disminución significativa de presión arterial sistólica de $24 \mathrm{~h}$ según monitoreo ambulatorio, a las 8 semanas de tratamiento $(\overline{\mathrm{x}}=-5,4 \mathrm{mmHg}, \mathrm{p}=0,024)^{61}$. El estudio no encontró diferencias significativas de la presión arterial diastólica, resultado atribuido al mayor número relativo de pacientes con HTA de predominio sistólico ${ }^{61}$. Al comparar fármacos antihipertensivos de cuarta línea, un estudio cruzado prospectivo ha mostrado que los ARM tendrían un mayor efecto antihipertensivo en pacientes con HTAR en comparación al doble bloqueo de angiotensina II utilizando un ARA2 $(-20,8 \pm-14,6 /-8,8 \pm-7,3$ vs $-7,1 \pm 13,4 /-3,4$ $\pm 6,2 \mathrm{mmHg}$ respectivamente, $\mathrm{n}=42)^{62}$. Estos resultados se explicarían debido a que los IECA/ ARA2 son incapaces de prevenir a largo plazo el aumento de concentración plasmática de aldosterona, fenómeno conocido como escape de aldosterona ${ }^{63}$.

Un reciente meta-análisis, que incluyó 5 estudios cruzados en pacientes con HTA esencial seguidos por 4-6 semanas, señaló que espironolactona reduce la presión arterial de forma significativa $(\overline{\mathbf{X}}=-20,01 /-6,75 \mathrm{mmHg})^{55}$. Lamentablemente, el efecto antihipertensivo requiere de dosis de $100-500 \mathrm{mg} /$ día, asociadas a un mayor riesgo de hiperkalemia ${ }^{55,56}$. Sin embargo, estudios en población hipertensa con dosis bajas de espironolactona añadido a IECA/ARA2 han señalado una mejora de la función ventricular izquierda ${ }^{26}$. Un ensayo clínico con distribución aleatoria en pacientes con HTA esencial $(n=409)$, comparando diversas dosis de eplerenona (50-400 mg/día) contra placebo y espironolactona (100 mg/día), reportó una reducción significativa de la presión arterial sistólica y diastólica en todos los grupos tratados con ARM versus placebo $(\mathrm{p}<0,05)^{57}$. El grupo tratado con eplerenona (100 mg/día) tuvo 
una menor disminución de presión arterial y una menor tasa de efectos adversos que el grupo con espironolactona $(100 \mathrm{mg} / \mathrm{día})^{57}$. El estudio con distribución aleatoria 4E-Left Ventricular Hypertrophy Study $(\mathrm{n}=202)$, demostró que eplerenona es una alternativa eficaz como monoterapia, teniendo un efecto antihipertensivo similar al enalapril $(-23,8 /-11,9 \text { vs }-24,7 /-13,4 \mathrm{mmHg})^{58}$. Además, eplerenona y enalapril lograron disminuir de forma comparable la hipertrofia ventricular izquierda y la albuminuria ${ }^{58}$. Lo anterior sugiere que los ARM podían ser de utilidad como segunda línea de tratamiento de HTA, particularmente enfocada en tratar sus complicaciones.

Por último, aun cuando no es posible abordar en esta revisión la relación entre aldosterona y síndrome metabólico es importante señalar que existe un mayor riesgo de enfermedades cardiovasculares en individuos que presentan este síndrome que se caracteriza, entre otros, por aldosterona elevadas con reninas normales. El exceso de aldosterona estaría mediado por adipoquinas secretadas por el tejido adiposo que serían potentes secretágogos de la hormona; a su vez la activación en adipocitos del MR altera la función del tejido adiposo favoreciendo la acumulación de grasas ${ }^{4,64}$.

\section{Conclusión}

La presente revisión ha pretendido recoger las recomendaciones actuales de las guías nacionales e internacionales respecto del uso de los ARM en patologías tales como el HAP, IC NYHA III-IV y post-IAM. Algunos expertos consideran que existiría una subutilización de los ARM en el manejo de pacientes con IC a nivel mundial, probablemente debido al temor de inducir hiperkalemia, en especial al combinarse con IECA o ARA2 $2^{9,10}$. A la hora de llevar a la práctica clínica cotidiana los resultados de estos trabajos, se hace evidente que los ensayos clínicos randomizados doble ciego, aun cuando incluyen un gran número de pacientes, emplean -y con razón- criterios restrictivos de inclusión y exclusión, lo que exige cautela a la hora de evaluar sus resultados y de aplicarlos a la realidad asistencial diaria. En este sentido vale la pena considerar la posibilidad de acudir a los diferentes resultados obtenidos a través de un sistema abierto de inclusión online como son los estudios ALBATROSS y TOPCAT.

\section{Referencias}

1. Fardella CE, Mosso LM, Carvajal CA. Hiperaldosteronismo primario. Rev Med Chile 2008; 136: 905-14.

2. Milliez P, Girerd X, Plouin PF, Blacher J, Safar ME, Mourad JJ. Evidence for an increased rate of cardiovascular events in patients with primary aldosteronism. J Am Coll Cardiol 2005; 45: 1243-8.

3. Acelajado MC, Calhoun DA. Aldosteronism and resistant hypertension. Int J Hypertens 2011; 2011 : 837817.

4. Bravo S, Marusic ET. Aldosterona, una hormona pluripotencial: efectos en el riñón, sistema cardiovascular, cerebro y síndrome metabólico. Rev Chil Endocrinol Diabetes 2011; 4: 205-12.

5. Nappi JM, Sieg A. Aldosterone and aldosterone receptor antagonists in patients with chronic heart failure. Vasc Health Risk Manag 2011; 7: 353-63.

6. Dickstein K, Cohen-Solal A, Filippatos G, McMurray JJ, Ponikowski P, Poole-Wilson PA, et al. ESC Guidelines for the diagnosis and treatment of acute and chronic heart failure 2008: the Task Force for the Diagnosis and Treatment of Acute and Chronic Heart Failure 2008 of the European Society of Cardiology. Developed in collaboration with the Heart Failure Association of the ESC (HFA) and endorsed by the European Society of Intensive Care Medicine (ESICM). Eur Heart J 2008; 29: 2388-442.

7. Hunt SA, Abraham WT, Chin MH, Feldman AM, Francis GS, Ganiats TG, et al. 2009 focused update incorporated into the ACC/AHA 2005 Guidelines for the Diagnosis and Management of Heart Failure in Adults: a report of the American College of Cardiology Foundation/American Heart Association Task Force on Practice Guidelines: developed in collaboration with the International Society for Heart and Lung Transplantation. Circulation 2009; 119: e391-479.

8. Juurlink DN, Mamdani MM, Lee DS, Kopp A, Austin PC, Laupacis A, et al. Rates of hyperkalemia after publication of the Randomized Aldactone Evaluation Study. N Engl J Med 2004; 351: 543-51.

9. Guglin M, Awad KE, Polavaram L, Vankayala H. Aldosterone antagonists: the most underutilized class of heart failure medications. Am J Cardiovasc Drugs 2007; 7: 75-9.

10. Samuel JL, Delcayre C. Heart failure: aldosterone antagonists are underused by clinicians. Nat Rev Cardiol 2010; 7: 125-7.

11. Pitt B, Zannad F, Remme WJ, Cody R, Castaigne A, Pérez $A$, et al. The effect of spironolactone on morbidity and mortality in patients with severe heart failure. Randomized Aldactone Evaluation Study Investigators. N Engl J Med 1999; 341: 709-17. 
12. Pitt B, Remme W, Zannad F, Neaton J, Martínez F, Roniker B, et al. Eplerenone, a selective aldosterone blocker, in patients with left ventricular dysfunction after myocardial infarction. N Engl J Med 2003; 348: 1309-21.

13. Ezekowitz JA, McAlister FA. Aldosterone blockade and left ventricular dysfunction: a systematic review of randomized clinical trials. Eur Heart J 2009; 30: 469-77.

14. Wei J, Ni J, Huang D, Chen M, Yan S, Peng Y. The effect of aldosterone antagonists for ventricular arrhythmia: a meta-analysis. Clin Cardiol 2010; 33: 572-7.

15. Kotlyar E, Vita JA, Winter MR, Awtry EH, Siwik DA, Keaney JF, Jr., et al. The relationship between aldosterone, oxidative stress, and inflammation in chronic, stable human heart failure. J Card Fail 2006; 12: 122-7.

16. Michea L, Villagrán A, Urzúa A, Kuntsmann S, Marusic ET. Mineralocorticoid receptor antagonism attenuates cardiac hypertrophy and prevents oxidative stress in uremic rats. Hypertension 2008; 52: 295-300.

17. Zannad F, Alla F, Dousset B, Pérez A, Pitt B. Limitation of excessive extracellular matrix turnover may contribute to survival benefit of spironolactone therapy in patients with congestive heart failure: insights from the randomized aldactone evaluation study (RALES). Rales Investigators. Circulation 2000; 102: 2700-6.

18. Iraqi W, Rossignol P, Angioi M, Fay R, Nuee J, Ketelslegers JM, et al. Extracellular cardiac matrix biomarkers in patients with acute myocardial infarction complicated by left ventricular dysfunction and heart failure: insights from the Eplerenone Post-Acute Myocardial Infarction Heart Failure Efficacy and Survival Study (EPHESUS) study. Circulation 2009; 119: 2471-9.

19. Zannad F, McMurray JJ, Krum H, van Veldhuisen DJ, Swedberg K, Shi H, et al. Eplerenone in patients with systolic heart failure and mild symptoms. N Engl J Med 2011; 364: 11-21.

20. Macdonald JE, Kennedy N, Struthers AD. Effects of spironolactone on endothelial function, vascular angiotensin converting enzyme activity, and other prognostic markers in patients with mild heart failure already taking optimal treatment. Heart 2004; 90: 765-70.

21. Udelson JE, Feldman AM, Greenberg B, Pitt B, Mukherjee R, Solomon HA, et al. Randomized, double-blind, multicenter, placebo-controlled study evaluating the effect of aldosterone antagonism with eplerenone on ventricular remodeling in patients with mild-tomoderate heart failure and left ventricular systolic dysfunction. Circ Heart Fail 2010; 3: 347-53.

22. Boccanelli A, Mureddu GF, Cacciatore G, Clemenza F, Di Lenarda A, Gavazzi A, et al. Anti-remodelling effect of canrenone in patients with mild chronic heart failure (AREA IN-CHF study): final results. Eur J Heart Fail
2009; 11: 68-76.

23. Vizzardi E, D’Aloia A, Giubbini R, Bordonali T, Bugatti $\mathrm{S}$, Pezzali N, et al. Effect of Spironolactone on Left Ventricular Ejection Fraction and Volumes in Patients With Class I or II Heart Failure. Am J Cardiol 2010; 106: 1292-6.

24. Ohtani T, Ohta M, Yamamoto K, Mano T, Sakata Y, Nishio $\mathrm{M}$, et al. Elevated cardiac tissue level of aldosterone and mineralocorticoid receptor in diastolic heart failure: Beneficial effects of mineralocorticoid receptor blocker. Am J Physiol Regul Integr Comp Physiol 2007; 292: R946-54.

25. Grandi AM, Imperiale D, Santillo R, Barlocco E, Bertolini A, Guasti L, et al. Aldosterone Antagonist Improves Diastolic Function in Essential Hypertension. Hypertension 2002; 40: 647-52.

26. Mottram PM, Haluska B, Leano R, Cowley D, Stowasser M, Marwick TH. Effect of Aldosterone Antagonism on Myocardial Dysfunction in Hypertensive Patients With Diastolic Heart Failure. Circulation 2004; 110: 558-65.

27. Daniel KR, Wells G, Stewart K, Moore B, Kitzman DW. Effect of aldosterone antagonism on exercise tolerance, Doppler diastolic function, and quality of life in older women with diastolic heart failure. Congest Heart Fail 2009; 15: 68-74.

28. Roongsritong C, Sutthiwan P, Bradley J, Simoni J, Power $\mathrm{S}$, Meyerrose GE. Spironolactone improves diastolic function in the elderly. Clin Cardiol 2005; 28: 484-7.

29. Deswal A, Richardson P, Bozkurt B, Mann DL. Results of the Randomized Aldosterone Antagonism in Heart Failure With Preserved Ejection Fraction Trial (RAAMPEF). J Card Fail 2011; 17: 634-42.

30. Desai AS, Lewis EF, Li R, Solomon SD, Assmann SF, Boineau R, et al. Rationale and design of the Treatment of Preserved Cardiac Function Heart Failure with an Aldosterone Antagonist Trial: A randomized, controlled study of spironolactone in patients with symptomatic heart failure and preserved ejection fraction. Am Heart J 2011; 162: 966-72 e10.

31. Edelmann F, Schmidt AG, Gelbrich G, Binder L, Herrmann-Lingen $\mathrm{C}$, Halle $\mathrm{M}$, et al. Rationale and design of the 'aldosterone receptor blockade in diastolic heart failure' trial: a double-blind, randomized, placebocontrolled, parallel group study to determine the effects of spironolactone on exercise capacity and diastolic function in patients with symptomatic diastolic heart failure (Aldo-DHF). Eur J Heart Fail 2010; 12: 874-82.

32. Schmidt K, Tissier R, Ghaleh B, Drogies T, Felix SB, Krieg T. Cardioprotective effects of mineralocorticoid receptor antagonists at reperfusion. Eur Heart J 2010; 31: 1655-62. 
33. Beygui F, Collet JP, Benoliel JJ, Vignolles N, Dumaine $\mathrm{R}$, Barthelemy $\mathrm{O}$, et al. High plasma aldosterone levels on admission are associated with death in patients presenting with acute ST-elevation myocardial infarction. Circulation 2006; 114: 2604-10.

34. Perrier E, Kerfant BG, Lalevee N, Bideaux P, Rossier MF, Richard S, et al. Mineralocorticoid receptor antagonism prevents the electrical remodeling that precedes cellular hypertrophy after myocardial infarction. Circulation 2004; 110: 776-83.

35. Fraccarollo D, Galuppo P, Schraut S, Kneitz S, van Rooijen N, Ertl G, et al. Immediate mineralocorticoid receptor blockade improves myocardial infarct healing by modulation of the inflammatory response. Hypertension 2008; 51: 905-14.

36. Hayashi M, Tsutamoto T, Wada A, Tsutsui T, Ishii C, Ohno $\mathrm{K}$, et al. Immediate administration of mineralocorticoid receptor antagonist spironolactone prevents post-infarct left ventricular remodeling associated with suppression of a marker of myocardial collagen synthesis in patients with first anterior acute myocardial infarction. Circulation 2003; 107: 2559-65.

37. Beygui F, Labbé J-P, Cayla G, Ennezat P-V, Motreff P, Roubille F, et al. Early mineralocorticoid receptor blockade in primary percutaneous coronary intervention for ST-elevation myocardial infarction is associated with a reduction of life-threatening ventricular arrhythmia. Int J Cardiol 2011.

38. Beygui F, Vicaut E, Ecollan P, Machecourt J, Van Belle E, Zannad F, et al. Rationale for an early aldosterone blockade in acute myocardial infarction and design of the ALBATROSS trial. Am Heart J 2010; 160: 642-8.

39. Nattel S. Therapeutic implications of atrial fibrillation mechanisms: can mechanistic insights be used to improve AF management? Cardiovasc Res 2002; 54: 347-60.

40. Reil J-C, Hohl M, Selejan S, Lipp P, Drautz F, Kazakow A, et al. Aldosterone promotes atrial fibrillation. Eur Heart J 2012; 33: 2098-108.

41. De-An P, Li L, Zhi-Yun X, Jin-Yu H, Zheng-Ming X, Min W, et al. Increased expression of mineralocorticoid receptor and 11 beta-hydroxysteroid dehydrogenase type 2 in human atria during atrial fibrillation. Clin Cardiol 2010; 33: 23-9.

42. Tsai CT, Chiang FT, Tseng CD, Hwang JJ, Kuo KT, Wu $\mathrm{CK}$, et al. Increased expression of mineralocorticoid receptor in human atrial fibrillation and a cellular model of atrial fibrillation. J Am Coll Cardiol 2010; 55: 758-70.

43. Berglund H, Boukter S, Theodorsson E, Vallin H, Edhag O. Raised plasma concentrations of atrial natriuretic peptide are independent of left atrial dimensions in patients with chronic atrial fibrillation. Br Heart J 1990;
64: 9-13.

44. Goette A, Hoffmanns P, Enayati W, Meltendorf U, Geller JC, Klein HU. Effect of successful electrical cardioversion on serum aldosterone in patients with persistent atrial fibrillation. Am J Cardiol 2001; 88: 906-9.

45. Wozakowska-Kaplon B, Bartkowiak R, Janiszewska G. A decrease in serum aldosterone level is associated with maintenance of sinus rhythm after successful cardioversion of atrial fibrillation. Pacing Clin Electrophysiol 2010; 33: 561-5.

46. Delcayre C, Swynghedauw B. Molecular mechanisms of myocardial remodeling. The role of aldosterone. J Mol Cell Cardiol 2002; 34: 1577-84.

47. Michea L, Villagrán A, Urzúa A, Kuntsmann S, Venegas P, Carrasco L, Marusic ET. Mineralocorticoid receptor antagonism attenuates cardiac hypertrophy and prevents oxidative stress in uremic rats. Hypertension 2008; 52 : 295-300.

48. Kimura S, Ito M, Tomita M, Hoyano M, Obata H, Ding $\mathrm{L}$, et al. Role of mineralocorticoid receptor on atrial structural remodeling and inducibility of atrial fibrillation in hypertensive rats. Hypertens Res 2011; 34: 584-91.

49. Zhao J, Li J, Li W, Li Y, Shan H, Gong Y, et al. Effects of spironolactone on atrial structural remodelling in a canine model of atrial fibrillation produced by prolonged atrial pacing. Br J Pharmacol 2010; 159: 1584-94.

50. Swedberg K, Zannad F, McMurray JJ, Krum H, van Veldhuisen DJ, Shi H, et al. Eplerenone and atrial fibrillation in mild systolic heart failure: results from the EMPHASIS-HF (Eplerenone in Mild Patients Hospitalization And SurvIval Study in Heart Failure) study. J Am Coll Cardiol 2012; 59: 1598-603.

51. Williams RS, Delemos JA, Dimas V, Reisch J, Hill JA, Naseem RH. Effect of Spironolactone on Patients With Atrial Fibrillation and Structural Heart Disease. Clin Cardiol 2011; 34: 415-9.

52. Dabrowski R, Borowiec A, Smolis-Bak E, Kowalik I, Sosnowski C, Kraska A, et al. Effect of combined spironolactone-beta-blocker $+/$ - enalapril treatment on occurrence of symptomatic atrial fibrillation episodes in patients with a history of paroxysmal atrial fibrillation (SPIR-AF study). Am J Cardiol 2010; 106: 1609-14.

53. Vasan RS, Evans JC, Larson MG, Wilson PW, Meigs JB, Rifai N, et al. Serum aldosterone and the incidence of hypertension in nonhypertensive persons. $\mathrm{N}$ Engl J Med 2004; 351: 33-41.

54. Shibata H, Itoh H. Mineralocorticoid receptor-associated hypertension and its organ damage: clinical relevance for resistant hypertension. Am J Hypertens 2012; 25: 514-23. 
55. Batterink J, Stabler SN, Tejani AM, Fowkes CT. Spironolactone for hypertension. Cochrane Database Syst Rev 2010 (8): CD008169.

56. Parthasarathy HK, Menard J, White WB, Young WF, Jr., Williams GH, Williams B, et al. A double-blind, randomized study comparing the antihypertensive effect of eplerenone and spironolactone in patients with hypertension and evidence of primary aldosteronism. J Hypertens 2011; 29: 980-90.

57. Weinberger MH, Roniker B, Krause SL, Weiss RJ. Eplerenone, a selective aldosterone blocker, in mildto-moderate hypertension. Am J Hypertens. 2002; 15: 709-16.

58. Pitt B, Reichek N, Willenbrock R, Zannad F, Phillips RA, Roniker B, et al. Effects of eplerenone, enalapril, and eplerenone/enalapril in patients with essential hypertension and left ventricular hypertrophy: the $4 \mathrm{E}-\mathrm{left}$ ventricular hypertrophy study. Circulation 2003; 108: 1831-8.

59. Marrs JC. Spironolactone management of resistant hy- pertension. Ann Pharmacother 2010; 44: 1762-9.

60. Calhoun DA, White WB. Effectiveness of the selective aldosterone blocker, eplerenone, in patients with resistant hypertension. J Am Soc Hypertens 2008; 2: 462-8.

61. Vaclavik J, Sedlak R, Plachy M, Navratil K, Plasek J, Jarkovsky J, et al. Addition of Spironolactone in Patients With Resistant Arterial Hypertension (ASPIRANT): A Randomized, Double-Blind, Placebo-Controlled Trial. Hypertension 2011; 57: 1069-75.

62. Álvarez-Álvarez B, Abad-Cardiel M, Fernández-Cruz A, Martell-Claros N. Management of resistant arterial hypertension: role of spironolactone versus double blockade of the renin-angiotensin-aldosterone system. J Hypertens 2010; 28: 2329-35.

63. Bomback AS, Klemmer PJ. The incidence and implications of aldosterone breakthrough. Nat Clin Pract Nephrol 2007; 3: 486-92.

64. Briet M, Schiffrin EL.The role of aldosterone in the metabolic syndrome. Curr Hypertens Rep 2011; 13: 163-72 (review). 